\title{
The Value and the Variability of Magnetic Resonance Imaging in Patients with Systemic Lupus Erythematosus and Antiphospholipid Syndrome
}

\author{
Ekaterini Solomou, Aspasia Rigopoulou, Theodoros Petsas \\ Radiology Department, University Hospital of Patras, Patras, Greece \\ Email: aspasrigop@yahoo.gr
}

Received 24 January 2015; accepted 22 March 2015; published 26 March 2015

Copyright (C) 2015 by authors and Scientific Research Publishing Inc.

This work is licensed under the Creative Commons Attribution International License (CC BY).

http://creativecommons.org/licenses/by/4.0/

(c) (i) Open Access

\begin{abstract}
Objectives: The objectives of this article are twofold: To review the value and the variability of MR imaging (MRI) findings in patients with systemic lupus erythematosus (SLE) and to reveal the frequency of central nervous system (CNS) involvement. SLE is a complex multisystem autoimmune connective tissue disorder with a broad spectrum of clinical presentations. Materials and methods: We present three cases, two with neurological symptoms and one case with musculoskeletal symptoms. Patients were imaged in the MRI department of the University Hospital of Patras. All patients had a previous history of antiphospholipid syndrome and known SLE. Results: None of the pts had a normal brain MRI. Abnormal lesions were typically high on FLAIR and T2-weighted images. One patient showed myositis of the tibial muscles bilateral and although she did not reveal any neurological symptoms, because of the presence of APS, she underwent brain MRI to detect any cerebrovascular involvement. The brain MRI was abnormal and showed extensive lesions and porencephalia. In all cases the differential diagnosis of the brain MRI included vasculitis, focal ischemia, multiple sclerosis or other entities. Parenchymal volume loss-cerebral atrophy, incompatible with their age, was obvious in all patients. Conclusion: Magnetic resonance imaging is the gold standard for the investigation of central nervous system in patients suffering from lupus. Lupus patients who also develop antiphospholipid syndrome must be submitted to brain MRI because central nervous involvement is very common and serious, although symptoms may be silent as in our case.
\end{abstract}

\section{Keywords}

Systemic Lupus Erythematosus, Antiphospholipid Syndrome, MRI, Brain MRI 


\section{Introduction}

SLE is a complex multisystem autoimmune connective tissue disorder with a broad spectrum of clinical presentations. It affects almost all organ systems and tissues [1]. Neurologic and musculoskeletal manifestations are among its features. Central nervous system (CNS) lupus is a serious illness, which should be in the differential diagnosis for many neurologic conditions.

Patients who have antiphospholipid antibodies (APL-Ab) are at additional risk for central nervous involvement and neuropsychiatric event [2] [3].

Musculoskeletal involvement in lupus patients is common with characteristic manifestations, especially symmetric polyarthritis, deforming non-erosive arthropathy, myositis, osteomyelitis, osteonecrosis and septic arthritis [4].

\section{Materials and Methods}

$3 \mathrm{~F}$ patients in the age range of 23 to 40 years old were imaged in the MRI department of the University Hospital of Patras during the period 2011-2014. All patients, had a previous history of antiphospholipid syndrome and known SLE, confirmed by biopsy.

2 patients with neurological symptoms complained for headaches and seizures whereas the last one presented with nusculoskeletal symptoms—swollen tibias—and without any neurological symptoms.

All pts underwent brain MRI, independently of the presence of symptoms. The latter with MSK manifestations underwent also MRI for the lower extremities.

All examinations were performed at 1.5 Tesla MR.

The MRI of the brain included T1, T2 and Fluid-attenuated inversion-recovery (FLAIR) weighted images, including three planes. Gadolinium (GD) was injected in 2 of the 3 pts, because of the presence of SLE nephritis in 1 case.

All MRI studies were reviewed in consensus by two radiologists.

Images were studied for loss of brain volume, brain lesions with abnormal signal and pathologic contrast enhancement.

Brain lesions were classified as infarct-like with diameter $>10 \mathrm{~mm}$ or white-matter lesions , subcortical, deep or periventricular $<10 \mathrm{~mm}$. The site and number of the lesions were also noted.

The MR protocol for the musculoskeletal contains T1, T2, Fat Saturated (STIR) Wi at least in two planes and after GD administration T1 with Fat Saturation.

\section{Results}

Clinical data extracted included age, sex, symptoms and imaging findings all of which are summarized in Table 1 .

None of the pts had a normal brain MRI.

Abnormal lesions were typically high on FLAIR and T2-weighted images, most frequently in the subcortical and deep white matter in the frontal and parietal lobes.

The first patient showed multiple lesions on both cerebral hemispheres, in the periventricular and subcortical white matter, in the splenium and on the left temporal lobe. Gadolinium was not used because of the presence of SLE nephritis (Figure 1). The DD could include MS or vascular disease.

The second patient with seizures revealed small focal lesions less than $1 \mathrm{~mm}$ bilateral in the periventricular and subcortical white matter with intense enhancement on the left parietal and right frontal lobe (Figure 2).

The last patient underwent both lower extremities MRI which showed myositis of the tibial muscles bilateral (Figure 3). Although this pt did not reveal any neurological symptoms, because of the presence of APS, she underwent brain MRI to detect possible cerebrovascular involvement. The brain MRI was abnormal with subcortical lesions extended to the cortex in the left parietal lobe, focal lesions in the deep white matter bilateral, as well as porencephalic lesions with peripheral gliosis on the left parietal lobe, compatible with old infarct. No pathologic enhancement was noticed (Figure 4).

In all cases the $\mathrm{D} / \mathrm{d}$ could include vasculitis, focal ischemia, multiple sclerosis or other entities.

Parenchymal volume loss-cerebral atrophy, incompatible with their age, was obvious in all patients.

Subarachnoid or parenchymal hemorrhage, subdural hematoma or intraparenchymal hemorrhage was not de- 

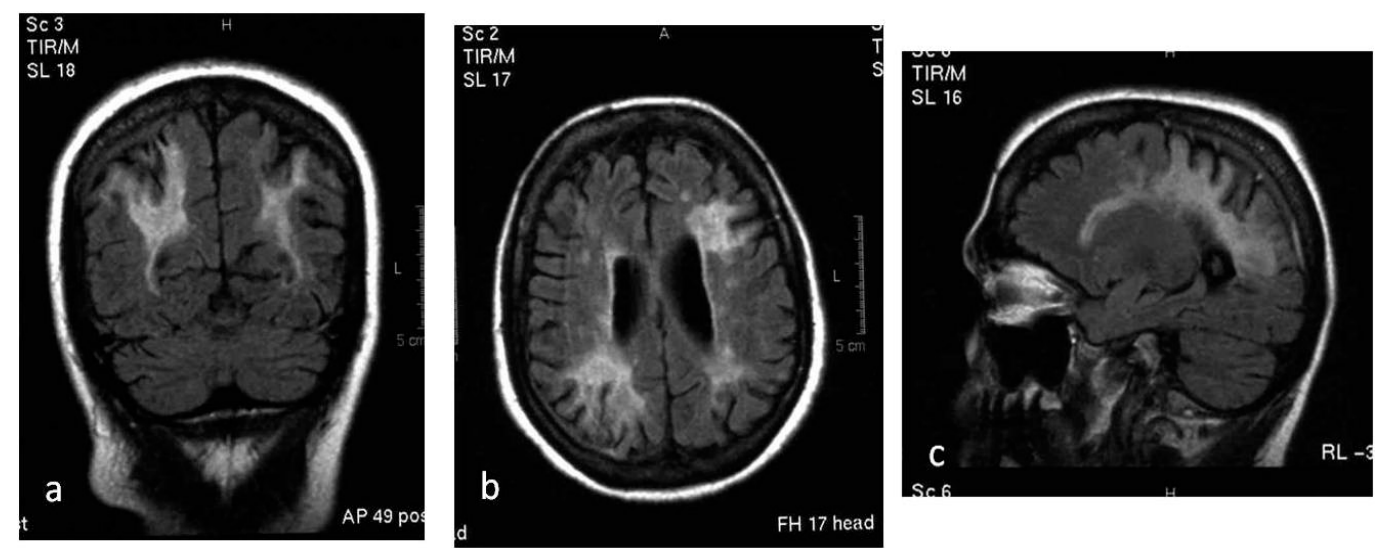

Figure 1. (a) Coronal, (b) axial and (c) Sagital FLAIR sequences. MRI shows symmetrical extensive hyperintensity lesions in the periventricular, deep and subcortical white matter, also involving the splenium. Brain atrophy is incompatible with the patient's age.
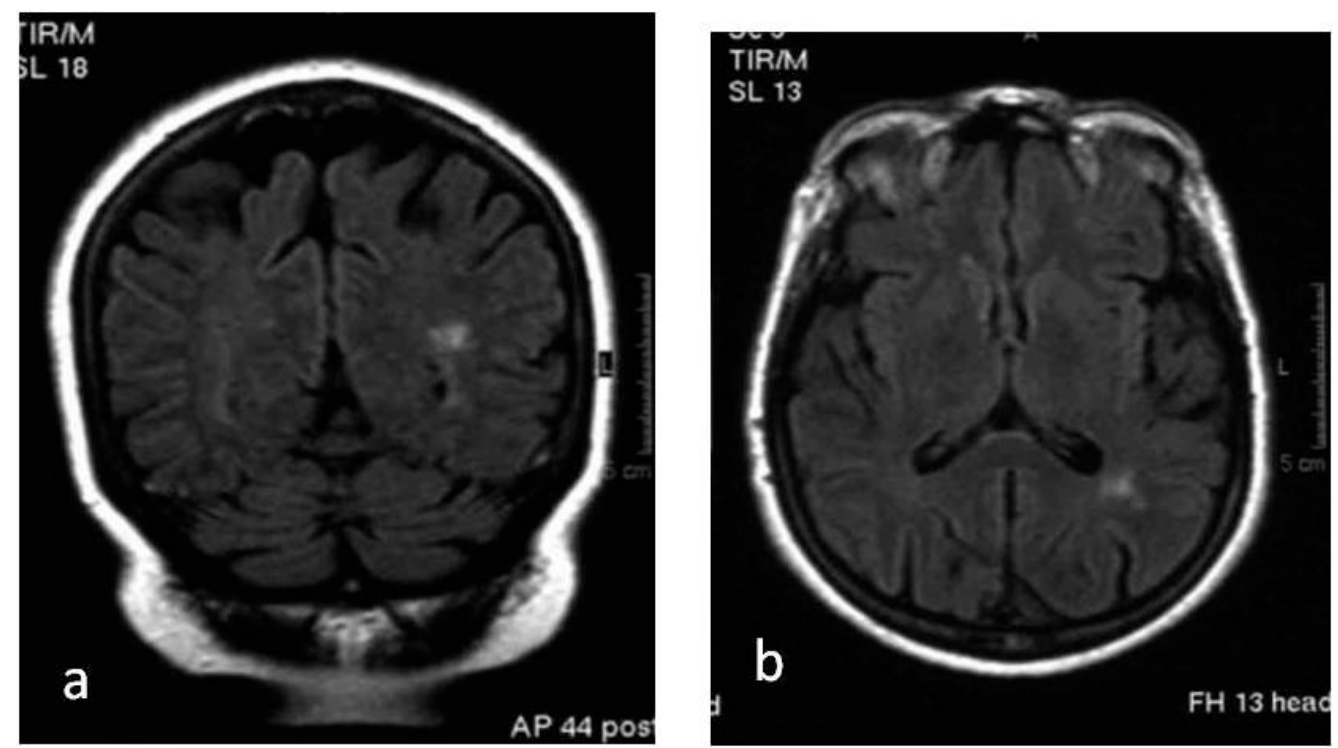

Figure 2. (a) Coronal, (b) axial FLAIR sequences show small lesions adjacent to the left occipital horn.

Table 1. Sex, age, symptoms and imaging findings.

\begin{tabular}{|c|c|c|c|c|}
\hline PT & TENDER/AGE & SYMPTOMS & MRI BRAIN & MRI TIBIA \\
\hline 1 & $\mathrm{~F} / 40$ & Headache & $\begin{array}{l}\text {-Multiple lesions } \\
\text {-Bilateral, } \\
\text {-Periventricular and subcortical white matter } \\
\text {-Splenium and } \\
\text {-Left temporal lobe_-Gd (-) }\end{array}$ & \\
\hline 2 & $\mathrm{~F} / 23$ & seizures & $\begin{array}{l}\text {-<1 mm focal lesions } \\
\text {-Bilateral } \\
\text {-Periventricular and subcortical white matter } \\
\text {-Gd: enhancement in the subcortical white matter on the left } \\
\text { Parietal and right frontal lobe }\end{array}$ & \\
\hline 3 & F/38 & $\begin{array}{l}\text { Swollen tibias } \\
\text { No neurological }\end{array}$ & $\begin{array}{l}\text {-Subcortical lesions extended to the cortex in the left parietal lobe, } \\
\text {-Focal lesions in the deep white matter bilateral, } \\
\text {-Porencephalic lesions with peripheral gliosis on the left parietal lobe } \\
\text {-Gd: no pathologic enhancement }\end{array}$ & $\begin{array}{l}\text { Myositis of the tibial } \\
\text { muscles bilateral }\end{array}$ \\
\hline
\end{tabular}




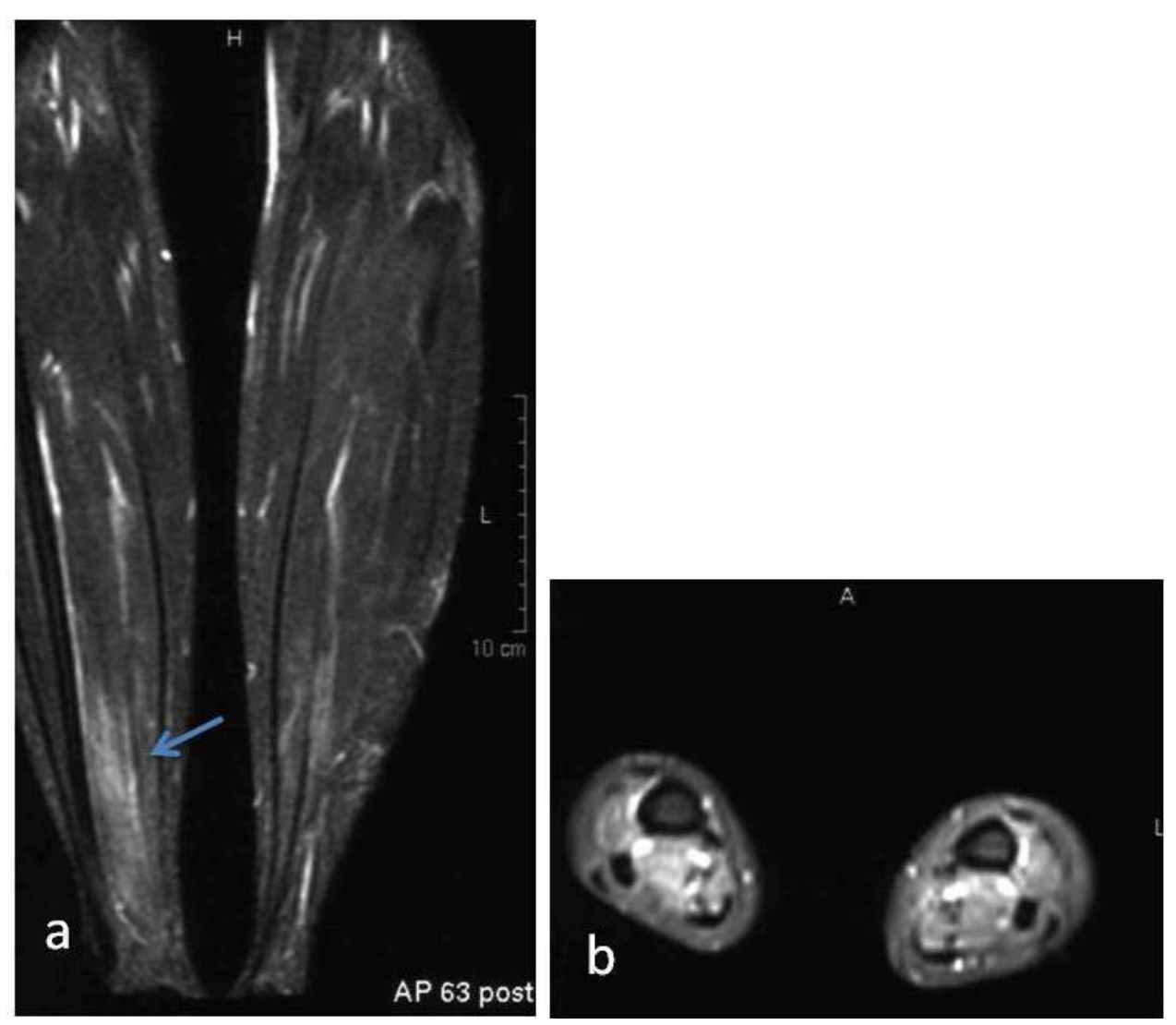

Figure 3. (a) Sagital, (b) axial MR Fat-Saturation images show (a) diffuse hyperintensity within the tibial muscles (arrow) with mild post contrast enhancement (b), findings consisted with myositis.
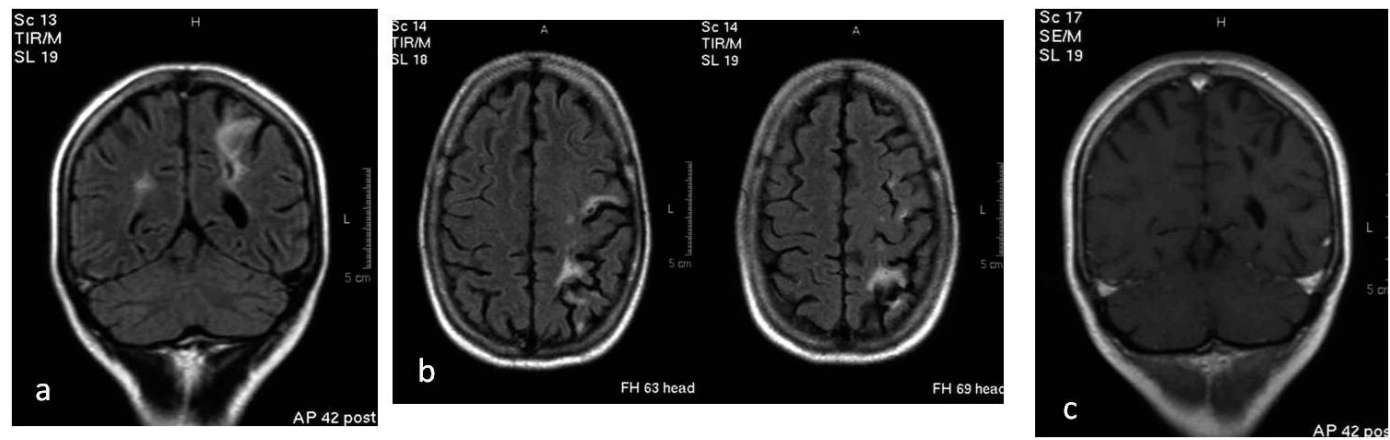

Figure 4. Same patient with Figure 3. (a) Coronal FLAIR sequence shows a porencephalic lesion with peripheral gliosis on the left frontal lobe without contrast enhancement (c). (b) Axial FLAIR sequence shows hyperintensity lesions in the subcortical matter of the left hemisphere.

tected in our study.

All findings were compatible and related to the underlying disease.

\section{Discussion}

Central nervous system (CNS) lupus is a serious illness, which is in the differential diagnosis for many neurologic conditions. Thus, clinicians need to be aware of the various presentations and neurologic complications of this illness; patients suffering from lupus often have neurologic symptoms while SLE is sometimes diagnosed after the presentation of a neurologic event [5] [6].

Neuropsychiatric systemic lupus erythematosus (NPSLE) occurs in 14\% - 75\% of cases, associated with an 
increased risk of death [7].

Patients with diffuse NPSLE may reveal lesions on brain MRI, even if they do not present any focal neurological manifestations [8].

The presence of brain MRI abnormalities does not affect the rate of relapse of SLE incidences, but it significantly increases mortality [9].

Approximately $40 \%$ of the NPSLE manifestations develop before the onset of SLE or at the time of diagnosis while $63 \%$ develop within the first year after the diagnosis [10].

An ischemic stroke can be isolated or multiple and recurrent.

Primary antiphospholipid syndrome (APS) is characterized by antiphospholipid antibodies (aPL) and significant thrombotic risk, including both deep venous and arterial thrombosis as well as spontaneous abortions. Secondary APS is commonly related to SLE. The coexistence of APS and SLE, as is the case with the patients in the present study, is relatively common and significantly increases the cerebrovascular injuries, causing headache, cognitive dysfunction and seizures but also strokes and transient ischemic attacks, mainly due to vessel occlusion [11] [12]. Recurrent stroke episodes are increased in APS patients after the first cerebral ischemic episode while the findings and the symptoms are not-dependent on the patient's age.

Although patients may also present other than neurological symptoms, as the last one in the present study, they also may have abnormal cerebrovascular findings on the imaging.

Depending on the size of the vessels involved, the brain MRI reveals lesions such as large or cortical infarcts, more often in the middle cerebral artery territory, with transient or permanent cerebral episodes in any vascular territory, porencephalic infarcts in the deep white matter bilateral and borderzone infarcts.

Cerebral alterations can be the result of microvascular disease, inflammation, antineuronal antibodies and treatment's side effects.

Atrophy can indeed reflect neuronal damage induced by SLE.

Lupus patients must always be submitted to brain MRI because central nervous involvement is very common and serious, although symptoms may be silent. In our patients, even with the absence of neurological symptoms, the most common abnormalities shown were cerebral atrophy, subcortical lesions and infracts, which are more common in pts with NPSLE according to the literature.

Musculoskeletal involvement in lupus patients [13] is common with characteristic manifestations in approximately $80 \%$. Symmetric polyarthritis, deforming non-erosive artrhopathy, myositis, osteomyelitis, osteonecrosis and septic arthritis are common because these patients are at increased risk of infections, due to steroid administration and renal disease.

Symmetric polyarthritis with varying degrees of severity is the most common clinical symptom, which is more severe in the morning. It usually affects the small joints of hand, knees, wrists and shoulders.

Deforming non-erosive arthropathy is present with long-standing disease as consequence of articular abnormalities in $5 \%-40 \%$ of the patients due to ligamentous laxity and muscle contracture.

Myositis can be observed in 30\% - 50\% of patients, but, clinically observed true myositis occurs in approximately only $4 \%$ of patients.

Osteonecrosis can be presented anywhere, but femoral head is most affected. This is more commonly seen in young patients, and those with Raynaud's phenomenon and other signs of vasculitis or as a complication of steroid therapy.

Osteomyelitis and septic arthritis can be present especially due to steroid administration and renal disease. Thus lupus patients are at increased risk of bacterial and mycotic infections, in large part. Bone and joint involvement is less common than infection elsewhere.

Other complications that may be seen in SLE are spontaneous tendon weakening and rupture, linear or nodular calcification in the subcutaneous and deep soft tissues, especially in the lower extremities, insufficiency fractures, possibly due to disuse demineralization or osteopenia secondary to steroid therapy, or both.

Spinal manifestations are uncommon and nonspecific, but a higher incidence of spinal findings is seen in those with deforming arthropathy.

\section{Conclusions}

Magnetic resonance imaging is the gold standard for the investigation of central nervous system in patients suffering from lupus. The most sensitive sequences are T2 and Flair, as they can visualize with bright signal edema 
or water, in contrary with T1 wi which may be normal.

Lupus usually coexists with antiphospholipid syndrome and those patients must always be submitted to brain MRI because central nervous involvement is very common and serious, although symptoms may be silent as in our case.

\section{References}

[1] Ruiz-Irastorza, G., Khamashta, M.A. and Castellino, G. (2001) Systemic Lupus Erythematosus. Lancet, 357, 10271032. http://dx.doi.org/10.1016/S0140-6736(00)04239-2

[2] Welsh, R.C., Rahbar, H., Foerster, B., Thurnher, M. and Sundgren, P.C. (2007) Brain Diffusivity in Patients with Neuropsychiatric Systemic Lupus Erythematosus with New Acute Neurological Symptoms. Journal of Magnetic Resonance Imaging, 26, 541-551. http://dx.doi.org/10.1002/jmri.21036

[3] Bertsias, G.K., Ioannidis, J.P. and Aringer, M. (2010) EULAR Recommendations for the Management of Systemic Lupus Erythematosus with Neuropsychiatric Manifestations: Report of a Task Force of the EULAR Standing Committee for Clinical Affairs. Annals of the Rheumatic Diseases, 69, 2074-2082. http://dx.doi.org/10.1136/ard.2010.130476

[4] Lalani, T.A., Kanne, J.P. and Hatfield, G.A. (2004) Imaging Findings in Systemic Lupus Erythematosus. Radiographics, 24, 1069-1086. http://dx.doi.org/10.1148/rg.244985082

[5] Hawro, T., Bogucki, A., Sysa-Jedrzejowska, A., Bogaczewicz, J. and Wozniacka, A. (2009) Neurological Disorders in Systemic Lupus Erythematosus Patients. Polski Merkuriusz Lekarski, 26, 43-48.

[6] Greenberg, B.M. (2009) The Neurologic Manifestations of Systemic Lupus Erythematosus. Neurologist, 15, $115-121$. http://dx.doi.org/10.1097/NRL.0b013e31818ff9aa

[7] Hanly, J.G., Cassell, K. and Kiska, J.D. (1997) Cognitive Function in Systemic Lupus Erythematosus: Results of a Five Year Prospective Study. Arthritis \& Rheumatism, 40, 1542-1543. http://dx.doi.org/10.1002/art.1780400825

[8] Castellino, G., Padovan, M., Bortoluzzi, A., Borrelli, M., Feggi, L., Caniatti, M.L., Trotta, F. and Govoni, M. (2008) Single Photon Emission Computed Tomography and Magnetic Resonance Imaging Evaluation in SLE Patients with and without Neuropsychiatric Involvement. Rheumatology, 47, 319-323. http://dx.doi.org/10.1093/rheumatology/kem354

[9] Arinuma, Y., Kikuchi, H., Wada, T., et al. (2014) Brain MRI in Patients with Diffuse Psychiatric/Neuropsychological Syndromes in Systemic Lupus Erythematosus. Lupus Science \& Medicine, 1, Article ID: e000050.

[10] Hanly, J.G., Urowitz, M.B. and Sanchez-Guerrero, J., et al. (2007) Neuropsychiatric Events at the Time of Diagnosis of Systemic Lupus Erythematosus: An International Inception Cohort Study. Arthritis \& Rheumatism, 56, 265-273. http://dx.doi.org/10.1002/art.22305

[11] Afeltra, A., Garzia, P., Mitterhofer, A.P., et al. (2003) Neuropsychiatric Lupus Syndromes: Relationship with Antiphospholipid Antibodies. Neurology, 61, 108-110. http://dx.doi.org/10.1212/01.WNL.0000058904.94330.A7

[12] Alarcón-Segovia, D., Delezé, M. and Oria, C.V. (1989) Antiphospholipid Antibodies and the Antiphospholipid Syndrome in Systemic Lupus Erythematosus. A Prospective Analysis of 500 Consecutive Patients. Medicine, 68, 353-365.

[13] Wallace, D.J. (2002) The Musculoskeletal System. In: Wallace, D.J. and Hahn, B.H., Eds., Dubois, Lupus Erythematosus, 6th Edition, Williams and Wilkins, Philadelphia, 629-644. 